\title{
Volatile Compound Profiles of Malus baccata and Malus prunifolia Wild Apple Fruit
}

\author{
Chunyu Zhang ${ }^{1}$ \\ College of Plant Science, Jilin University, Changchun, Jilin 130062, China \\ Xuesen Chen
National Key Laboratory of Crop Biology, Shandong Agricultural University, Taian, Shandong
271018 , China
}

Hongwei Song, Yinghai Liang, Chenhui Zhao, and Honglian Li
Pomology Institute, Academy of Agricultural Sciences of Jilin Province, Gongzhuling, Jilin 136100,
China

AdDitional Index words. apple breeding, cold resistance, ester, $\alpha$-farnesene, terpene

\begin{abstract}
Volatile compounds have a tremendous impact on fruit quality. We evaluated the volatile compound profiles of ripening wild apple fruit (10 Malus baccata accessions and three Malus prunifolia accessions) in the National Field Genebank for Hardy Fruits at Gongzhuling, China. Alcohols, esters, aldehydes, terpenes, hydrocarbons, ethers, heterocycles, carboxylic acids, and ketones were detected in the M. baccata and M. prunifolia fruit, with the first four being the main volatile compounds present. Of the 92 volatiles detected, esters were the most diverse (49 compounds). This wide range of abundant volatile compounds suggests that $M$. prunifolia is a good resource for breeding apple cultivars with novel and interesting flavors. The $M$. baccata accession 'Zhaai Shandingzi' and the $M$. prunifolia accession 'Bai Haitang' had the widest range of volatile compounds and the highest volatile compound contents of the accessions examined, and will therefore be good breeding materials for developing commercial lines with enhanced flavor and for widening the genetic diversity. The number of different ester compounds present was significantly positively correlated $(r=0.877)$ with the cube root of the weight of an individual ripe fruit. Principal component analysis (PCA) showed that the contents of ester compounds could be used to distinguish between M. baccata and $M$. prunifolia species. Therefore, ester compounds could be used as a reference of parental choice in apple breeding.
\end{abstract}

Apple (Malus pumila) is a fruit crop of major economic importance. In worldwide apple breeding efforts, apple breeders typically intercross elite or commercial cultivars, such as Red Delicious, Golden Delicious, and Jonathan, which results in commercial apple cultivars with a narrow genetic base, low adaptability, and reduced resistance to biotic and abiotic stresses (Kumar et al., 2010; Noiton and Alspach, 1996). Therefore, there is a need to develop breeding strategies that broaden the genetic base, enhance resistance and tolerance to biotic and abiotic stresses, and improve fruit quality, including flavor (Forsline et al., 2010; Sedov and Serova, 2013). Wild apples, with their high genetic diversity and antioxidant activity, disease resistance, and stress tolerance, are important resources for apple breeding (Chen et al., 2007; Emeriewen et al., 2014; John et al., 2014; Jurick et al., 2011; Zhang et al., 2007, 2003). To collect and protect hardy fruit resources, the National Field Genebank for Hardy Fruits, Gongzhuling, China, was established at Jilin Province (Zhang et al., 2003). Phenotypic properties, biologic characteristics, cold resistance, disease resistance, and fruit quality have been described and

Received for publication 27 Oct. 2016. Accepted for publication 23 Feb. 2017. This work was supported by a Special Fund for Agro-scientific Research in the Public Interest (201303093).

C. Zhang and X. Chen designed the experiment, performed the study, and drafted the manuscript. H. Song and Y. Liang determined the volatile compound contents of fruits. C. Zhao and $\mathrm{H}$. Li analyzed data and helped to draft the manuscript. All of the authors read and approved the final version of this manuscript.

${ }^{1}$ Corresponding author. E-mail: chunyuzhang1979@126.com. evaluated in this nursery (Zhang, 2008). Malus baccata and Malus prunifolia are the hardiest wild apple species in this nursery and also the main wild cold hardy species in northern China. These cold hardy fruit can tolerate temperatures below $-42{ }^{\circ} \mathrm{C}$ (Li et al., 2015; Wang et al., 2013; Zhang et al., 2003), and their hardy rootstocks are widely used for apple grafting and apple breeding in northern China. M. baccata and $M$. prunifolia accessions with excellent traits were formed by human selection over many years, and were cultivated for their fruit and as rootstocks (Kuznetsova et al., 2010; Meng et al., 1997; Zhang, 2008).

Volatile components have a huge impact on fruit quality, and are thus an important factor in apple breeding (Laurens, 1999). In apple, volatile components are composed of esters, alcohols, aldehydes, ketones, and ethers (Dimick and Hoskin, 1983; Kakiuchi et al., 1986). Research by Kakiuchi et al. (1986) showed that esters are the most abundant flavor components in apple cultivars (Hatsuaki, Kogyoku, Golden Delicious, Mutsu, and Fuji). Similar results were found for 'Annurca' and 'Delicious' apple fruit (Mattheis et al., 1991; Scalzo et al., 2001). In wild apple, aldehydes, esters, and alcohols are the main volatile compounds (Li et al., 2008). In Malus sieversii, alcohols, aldehydes, and esters are the main volatile compounds (Chen et al., 2007). However, the volatile compounds present in M. baccata and M. prunifolia are unknown.

In this work, we identified the volatile compounds present in 10 accessions of $M$. baccata and three accessions of $M$. prunifolia in the National Field Genebank for Hardy 
Fruits. The results of this study represent a resource for selecting and breeding cultivars with a desired volatile phenotype.

\section{Materials and Methods}

Plant materials. Malus baccata and M. prunifolia trees were planted in the National Field Genebank for Hardy Fruits (lat. $43^{\circ} 50^{\prime} \mathrm{N}$, long. $124^{\circ} 82^{\prime} \mathrm{E}$ ). The average temperature for January is $-13.6{ }^{\circ} \mathrm{C}$ and extreme low temperature is $-38{ }^{\circ} \mathrm{C}$ for the nursery. The management level of the nursery is good and consistent. The fully ripened apple fruit with detectable fragrance and intrinsic color of $10 \mathrm{M}$. baccata accessions ('Duanba Shandingzi', 'Xiaosuanguo', 'Zhaai Shandingzi', 'Benyuandaguo Shandingzi', 'Benzaodaguo Shandingzi', 'No. 3 Shandingzi', 'No. 5 Shandingzi', 'No. 6 Shandingzi', 'No. 7 Shandingzi', and 'No. 8 Shandingzi') and three $M$. prunifolia accessions ('Bai Haitang', 'Xiaohuang Haitang', and 'Bian Haitang') were harvested between 20 Aug. 2014 and 20 Sept. 2014 (Fig. 1). Three trees were sampled for each accession and 50 fruit were harvested for each tree. Apple fruit were harvested one time from each tree, all with about equal conditions of maturity and of about the same size and stature. All the fruit from three trees of the same accession were mixed and divided into three groups, and each group was a biological replicate. The apples were packed and shipped overnight to the State Key Laboratory for Crop Biology at Shandong Agricultural University, Tai'an, China, for evaluation.

Static Headspace EXtraction. Ten to 50 apple fruit were rapidly cut into slices of about $3 \mathrm{~mm}$ wide and mixed. Six grams of sample was placed in $10 \mathrm{~mL}$ sample bottles, and $10 \mu \mathrm{L} 3$-nonanone $\left(0.4 \mathrm{mg} \cdot \mathrm{mL}^{-1}\right)$ was injected into each bottle and used as an internal standard. The sample bottles were then sealed using polytetrafluoroethylene butyl rubber cap isolation. A headspace sampler (PerkinElmer TurboMatrix 40 Trap; GenTech, Arcade, NY) was used to extract the volatile component of fruit and inject the sample. Samples were extracted after $30 \mathrm{~min}$ of incubation at $50{ }^{\circ} \mathrm{C}$, with the temperature of the static sample probe and transfer line being maintained at $80^{\circ} \mathrm{C}$, and then the sample bottle was pressured to $15 \mathrm{~Pa}$ for $5 \mathrm{~min}$. Then, $500 \mu \mathrm{L}$ of the volatile component was captured for analysis.
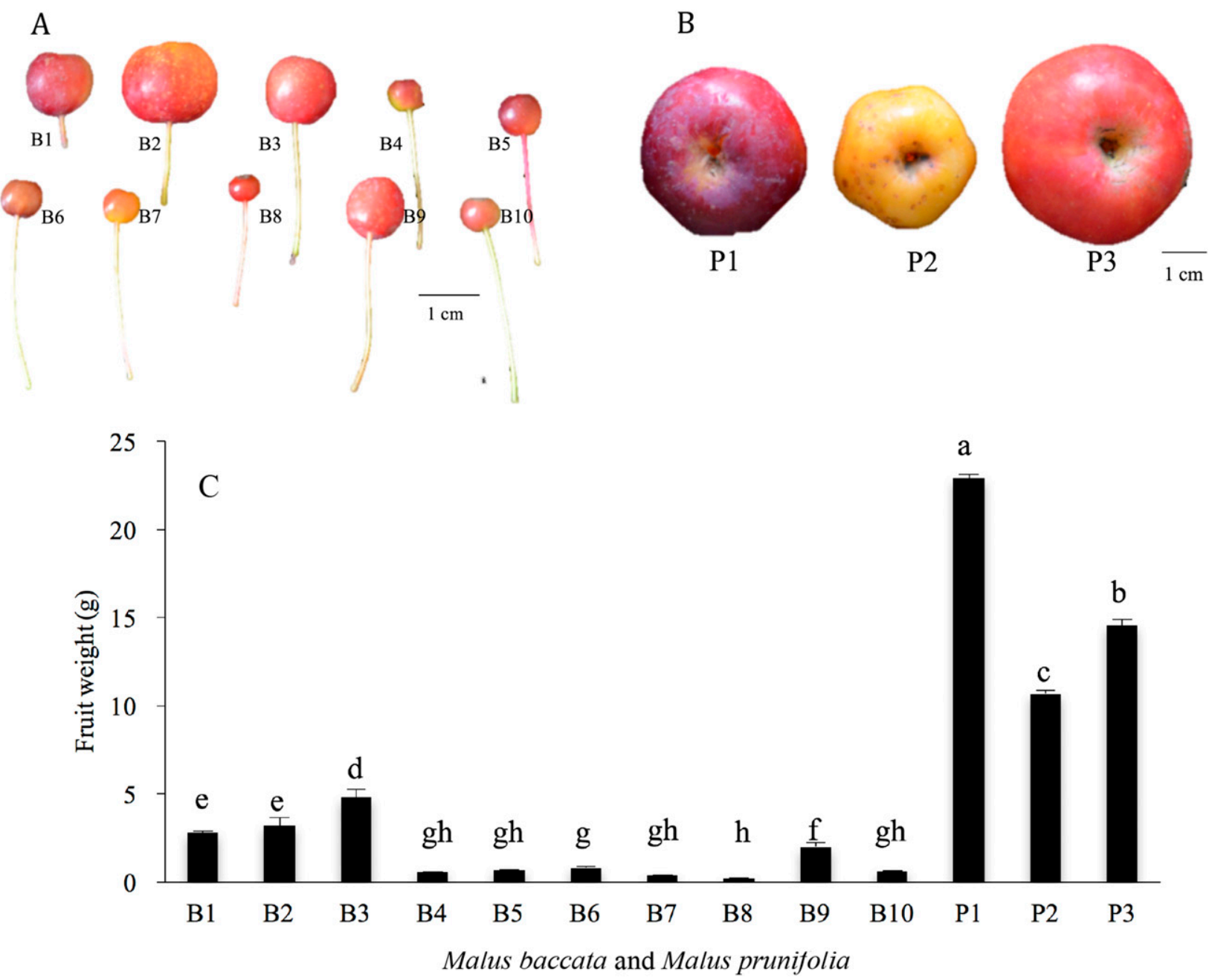

Fig. 1. Ripe fruit of (A) Malus baccata and (B) Malus prunifolia and (C) their weights. M. baccata accessions are abbreviated as follows: B1 = 'Duanba Shandingzi', B2 = 'Benyuandaguo Shandingzi', B3 = 'Benzaodaguo Shandingzi', B4 = 'No. 3 Shandingzi', B5 = 'No. 5 Shandingzi', B6 = 'No. 6 Shandingzi', B7 = 'No. 7 Shandingzi', B8 = 'No. 8 Shandingzi', B9 = 'Xiaosuanguo', and B10 = 'Zhaai Shandingzi'; M. prunifolia accessions are abbreviated as follows: P1 = 'Bai Haitang', P2 = 'Xiaohuang Haitang', and P3 = 'Bian Haitang'. The vertical bar represents \pm SE of the mean of 30 fruit. Different letters above the bars indicate significant differences at $P \leq 0.05$ based on Duncan's test. 


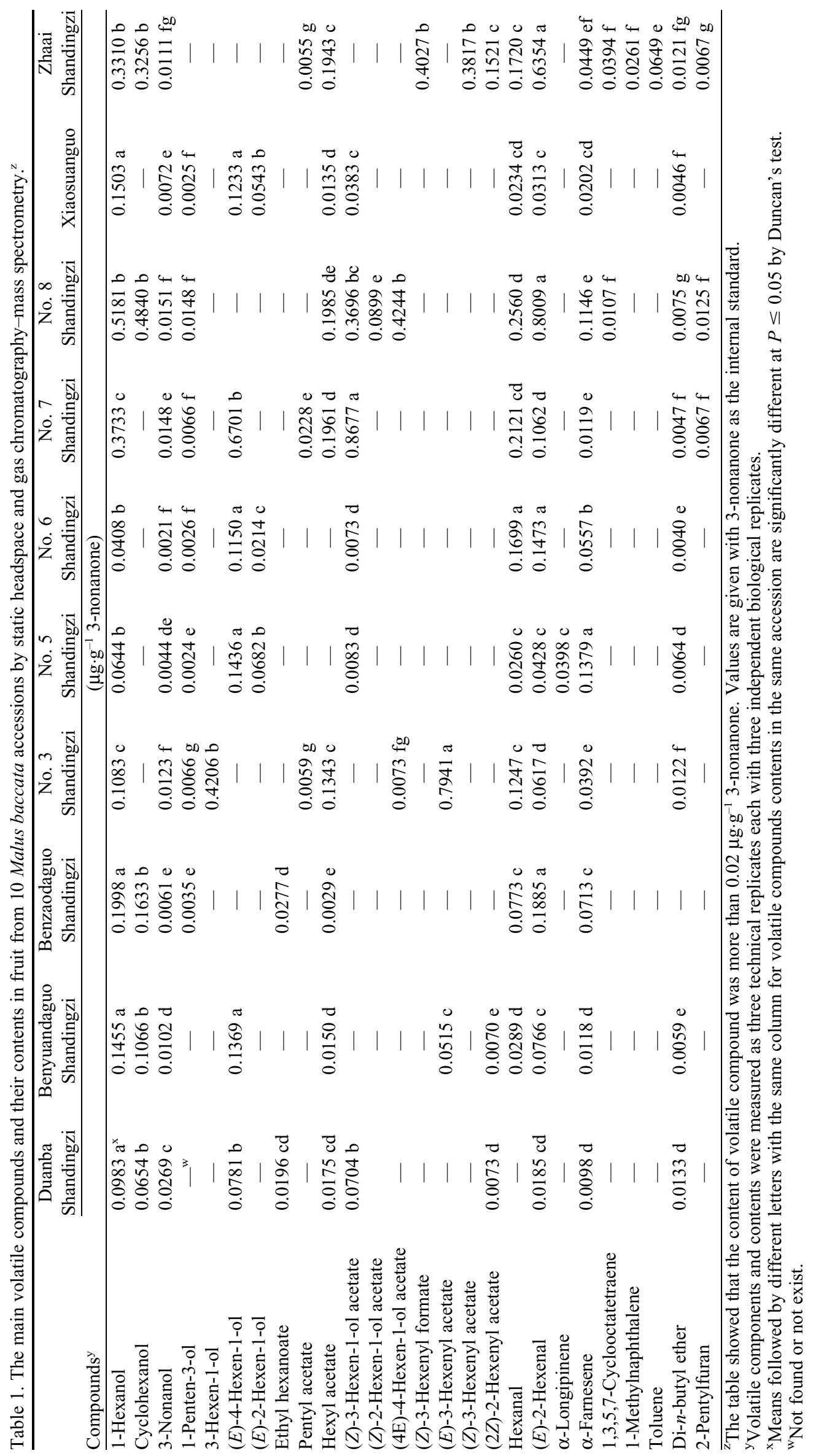


Table 2. The main volatile compounds and their contents in fruit from three Malus prunifolia accessions by static headspace and gas chromatography-mass spectrometry. ${ }^{z}$

\begin{tabular}{|c|c|c|c|}
\hline \multirow{2}{*}{ Compounds ${ }^{y}$} & Bai Haitang & Xiaohuang Haitang & Bian Haitang \\
\hline & \multicolumn{3}{|c|}{$\left(\mu \mathrm{g} \cdot \mathrm{g}^{-1}\right.$ 3-nonanone $)$} \\
\hline Cyclohexanol & $-^{x}$ & - & $0.0607 \mathrm{~d}$ \\
\hline 1-Hexanol & $0.1602 \mathrm{c}^{\mathrm{w}}$ & $0.2956 \mathrm{~b}$ & $0.1684 \mathrm{c}$ \\
\hline 1-Butanol & $0.0129 \mathrm{~g}$ & - & - \\
\hline 2-Methyl-1-butanol & 0.0303 ef & $0.0127 \mathrm{f}$ & - \\
\hline Methyl butanoate & $0.0465 \mathrm{e}$ & - & 0.0097 ef \\
\hline Methyl 2-methylbutanoate & $0.0573 \mathrm{~d}$ & - & $0.0250 \mathrm{e}$ \\
\hline Methyl hexanoate & $0.0689 \mathrm{~d}$ & - & $0.0239 \mathrm{e}$ \\
\hline Ethyl propionate & $0.0547 \mathrm{~d}$ & - & $0.0080 \mathrm{f}$ \\
\hline Ethyl hexanoate & $0.4518 \mathrm{~b}$ & $0.0662 \mathrm{c}$ & $0.2872 \mathrm{~b}$ \\
\hline Ethyl butanoate & $0.5837 \mathrm{a}$ & $0.0498 \mathrm{~d}$ & $0.1365 \mathrm{c}$ \\
\hline Ethyl 2-methylbutyrate & $0.4151 \mathrm{~b}$ & - & $0.1153 \mathrm{c}$ \\
\hline Ethyl tiglate & - & - & $0.0362 \mathrm{e}$ \\
\hline Propyl butyrate & $0.1138 \mathrm{c}$ & - & - \\
\hline Propyl hexanoate & $0.0488 \mathrm{~d}$ & - & - \\
\hline Propyl 2-methyl butyrate & $0.0699 \mathrm{~d}$ & - & - \\
\hline Butyl butyrate & $0.0527 \mathrm{~d}$ & - & - \\
\hline Butyl 2-methylbutyrate & $0.0483 \mathrm{e}$ & - & - \\
\hline Hexyl hexanoate & $0.0253 \mathrm{f}$ & $0.0039 \mathrm{~g}$ & $0.0064 \mathrm{f}$ \\
\hline Hexyl butyrate & $0.0578 \mathrm{~d}$ & $0.0403 \mathrm{~d}$ & $0.0211 \mathrm{e}$ \\
\hline (2Z)-2-Hexenyl acetate & - & $0.0053 \mathrm{~g}$ & - \\
\hline Hexyl acetate & - & $0.0241 \mathrm{e}$ & - \\
\hline Hexyl 2-methylbutyrate & $0.0412 \mathrm{e}$ & $0.0104 \mathrm{f}$ & 0.0119 ef \\
\hline (4E)-4-Hexenyl acetate & - & $0.0450 \mathrm{~d}$ & - \\
\hline Isopentyl 2-methylbutanoate & $0.0137 \mathrm{~g}$ & $0.0030 \mathrm{~g}$ & - \\
\hline (2E)-2-Hexenyl butyrate & $0.0098 \mathrm{~g}$ & $0.0233 \mathrm{e}$ & 0.0107 ef \\
\hline Hexanal & - & $0.0216 \mathrm{e}$ & - \\
\hline 2-Hexenal & - & $0.0769 \mathrm{c}$ & - \\
\hline$\alpha$-Curcumene & $0.0034 \mathrm{~h}$ & $0.0127 \mathrm{f}$ & 0.0138 ef \\
\hline$\alpha$-Farnesene & $0.5465 \mathrm{a}$ & $0.5262 \mathrm{a}$ & $0.4233 \mathrm{a}$ \\
\hline
\end{tabular}

${ }^{\mathrm{z}}$ The table showed that the content of volatile compound was more than $0.02 \mu \mathrm{g} \cdot \mathrm{g}^{-1}$ 3-nonanone. Values are given with 3-nonanone as the internal standard.

${ }^{\mathrm{y}}$ Volatile components and contents were measured as three technical replicates each with three independent biological replicates.

${ }^{\mathrm{x}}$ Not found or not exist.

${ }^{\mathrm{w}}$ Means followed by different letters with the same column for volatile compounds contents in the same accession are significantly different at $P \leq 0.05$ by Duncan's test.

GAS CHROMATOGRAPHY AND MASS SPECTROMETRY. A gas chromatography-mass spectrometry [GC-MS (GCMSQP2010; Shimadzu, Kyoto, Japan)] instrument was used to analyze the volatile composition of substances released from the apple slices. GC conditions were as follows: the chromatographic column used was Rtx-5 $(30 \mathrm{~m} \times 0.32 \mathrm{~mm} \times$ $1.0 \mu \mathrm{m}$; Restek, Bellefonte, PA); the injection port temperature was $200{ }^{\circ} \mathrm{C}$; and the column temperature was maintained at $35^{\circ} \mathrm{C}$ for $2 \mathrm{~min}$, at $8^{\circ} \mathrm{C} \cdot \mathrm{min}^{-1}$ to $180^{\circ} \mathrm{C}$ for $4 \mathrm{~min}$, and then at $15^{\circ} \mathrm{C} \cdot \mathrm{min}^{-1}$ to $230{ }^{\circ} \mathrm{C}$ for $1 \mathrm{~min}$. MS conditions were as follows: helium was used as the carrier gas at a flow rate of $1.06 \mathrm{~mL} \cdot \mathrm{min}^{-1}$. The $\mathrm{MS}$ was operated in the electron impact ionization mode with an electron energy of $70 \mathrm{eV}$. The scan range was 45-450 atomic mass units. Splitless injection of $1 \mu \mathrm{L}$ of solution was performed and the temperature of the ion source was $200{ }^{\circ} \mathrm{C}$.

Qualitative and Quantitative analysis. The GC data processing system, the computer retrieval and NIST library (107,000 compounds), and the Wiley library (y320 k com- pounds, version 6.0), combined with artificial map matching were used to confirm the chemical composition of volatile substances. Relative content was calculated according to the peak area normalization method (Wang et al., 2014). 3-Nonanone was used as an internal standard to estimate the volatile relative content.

Data analysis. Volatile components and contents were measured as three technical replicates each with three independent biological replicates. SPSS software (version 17.0; IBM Corp, Armonk, NY) was used for significant differences $(P \leq 0.05)$, PCA, and Pearson's correlation evaluation.

\section{Results}

Categorization of the main volatile COMPOUNDS RELEASED BY M. BACCATA AND $M$. PRUNIFOLIA FRUIT. We identified 58 and 54 volatile compounds, including alcohols, esters, aldehydes, terpenes, hydrocarbons, ethers, heterocycles, carboxylic acids, and ketones, in $10 \mathrm{M}$. baccata accessions and three M. prunifolia accessions using GC-MS (Supplemental Tables 1 and 2). The main volatile compounds identified (with contents of more than $0.02 \mu \mathrm{g} \cdot \mathrm{g}^{-1} 3$-nonanone) are shown in Tables 1 and 2 . We found that all $10 \mathrm{M}$. baccata accessions contained four common components: 1-hexanol, 3-nonanol, $(E)-2-$ hexenal, and $\alpha$-farnesene, with 1 -hexanol being the most abundant volatile component of 'Duanba Shandingzi', 'Benyuandaguo Shandingzi', 'Benzaodaguo Shandingzi', and 'Xiaosuanguo', and (E)-2-hexenal being the most abundant volatile component of 'No. 8 Shandingzi' and 'Zhaai Shandingzi'. (E)-3Hexenyl acetate, $(E)-4-h e x e n-1-o l$, hexanal, and (Z)-3-hexen-1-ol acetate were the most abundant volatile compounds present in 'No. 3 Shandingzi', 'No. 5 Shandingzi', 'No. 6 Shandingzi', and 'No. 7 Shandingzi', respectively (Table 1). In three $M$. prunifolia accessions, nine kinds of volatile compounds were common (Table 2), including 1-hexanol, ethyl hexanoate, ethyl butanoate, hexyl hexanoate, hexyl butyrate, hexyl 2-methylbutyrate, (2E)-2hexenyl butyrate, $\alpha$-curcumene, and $\alpha$-farnesene. For 'Bai Haitang', the content of ethyl butanoate $\left(0.5837 \mu \mathrm{g} \cdot \mathrm{g}^{-1} 3\right.$ nonanone) was highest among the volatile compounds, followed by $\alpha$-farnesene $\left(0.5465 \mu \mathrm{g} \cdot \mathrm{g}^{-1} 3\right.$-nonanone $)$. However, $\alpha$-farnesene was the most abundant volatile compound in 'Xiaohuang Haitang' $\left(0.5265 \mu \mathrm{g} \cdot \mathrm{g}^{-1}\right.$ 3-nonanone) and 'Bian Haitang' (0.4233 $\mu \mathrm{g} \cdot \mathrm{g}^{-1}$ 3-nonanone).

Furthermore, we determined the contents of alcohols, esters, aldehydes, terpenes, and hydrocarbons in M. baccata and $M$. prunifolia fruit (Fig. 2). For M. baccata accessions, the content of alcohols was highest in 'Duanba Shandongzi', 'Benyuandaguo Shandingzi', 'Benzaodaguo Shandingzi', 'No. 5 Shandingzi', and 'Xiaosuanguo' and the content of aldehydes was highest in 'No. 3 Shandingzi', 'No. 6 Shandingzi', 'No. 7 Shandingzi', 'No. 8 Shandingzi', and 'Zhaiai Shandingzi'. For the three M. prunifolia 


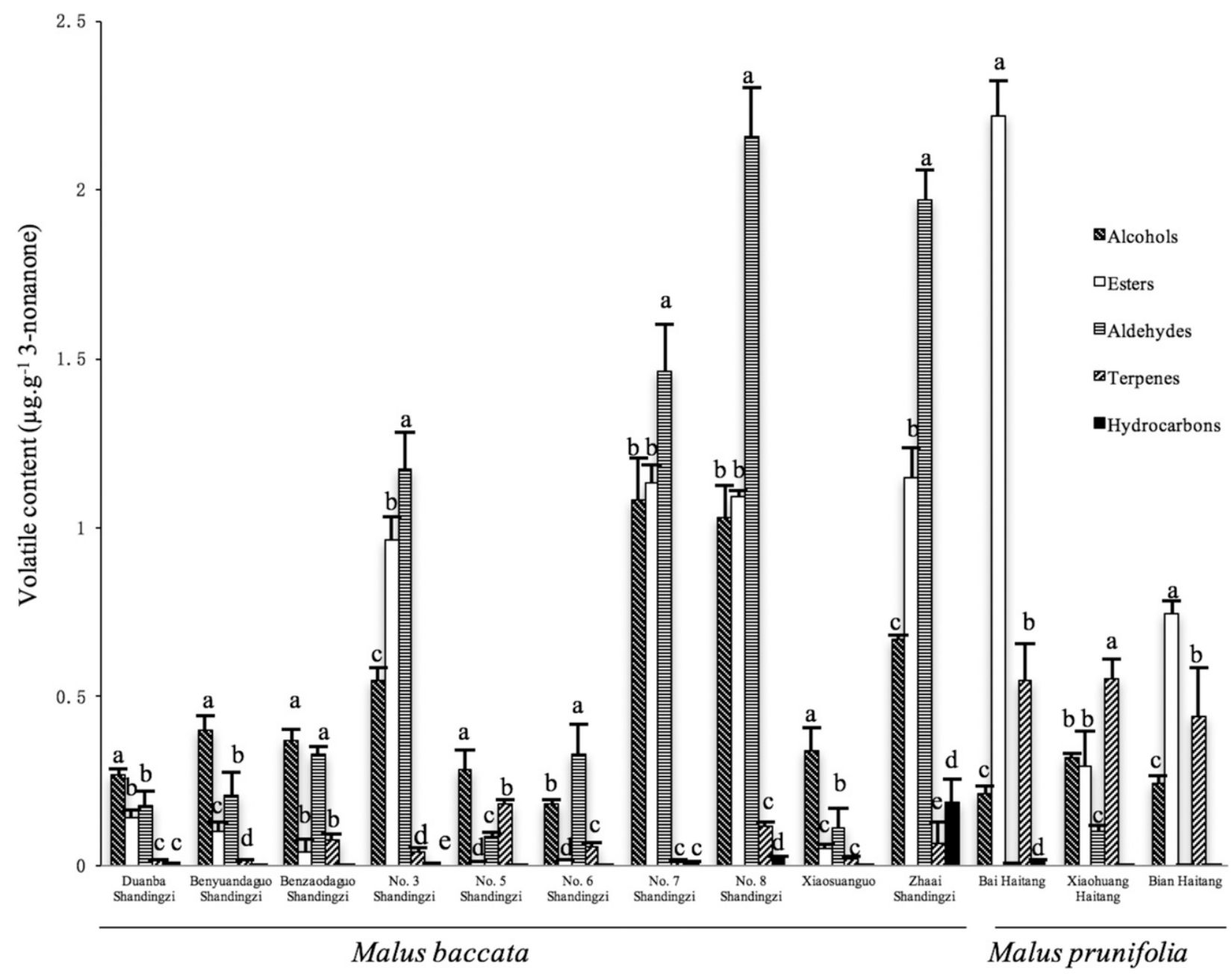

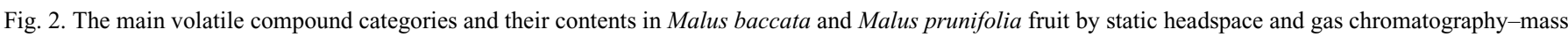
spectrometry. The values represent the mean of three independent biological replicates $( \pm \mathrm{SE})$. Different letters indicate significant differences among compound contents at same accession at $P \leq 0.05$ based on Duncan's test.

accessions examined, esters, terpenes, and alcohols were the most abundant volatile compounds present (Fig. 2).

VOLATILE COMPOUND TYPES AND CONTENTS IN $10 \mathrm{M}$. BACCATA AND 3 M. PRUNIFolia ACCESSIONS. In the 'Zhaai Shandingzi' accession, we detected 28 kinds of volatile compounds, 10 of which were hydrocarbons, 6 of which were esters, and 4 of which were aldehydes. 'Zhaai Shandingzi' was found to have the highest content of volatile compounds $\left(2.9096 \mu \mathrm{g} \cdot \mathrm{g}^{-1}\right.$ 3 -nonanone) among the $M$. baccata fruit (Table 3 and Supplemental Table 1). A total of 38,22 , and 25 kinds of volatile compounds were detected in 'Bai Haitang', 'Xiaohuang Haitang', and 'Bian Haitang' from M. prunifolia, respectively; most of which were esters, and 'Bai Haitang' had the highest contents of volatile compounds $\left(3.007 \mu \mathrm{g} \cdot \mathrm{g}^{-1}\right.$ 3-nonanone) among the $M$. prunifolia fruit (Table 3 and Supplemental Table 2). Esters (49 kinds) were the most diverse category of volatile compound, accounting for more than half of the total volatile compounds present ( $92 \mathrm{kinds}$ ).

RELATIONSHIP BETWEEN THE NUMBER OF VOLATILE COMPOUNDS AND FRUIT WEIGHT. To establish if fruit weight affects the number of volatile compounds, we conducted a correlation analysis between the volatile compounds and the cube root of the weight of an individual ripe fruit. As shown in
Fig. 3, the number of different types of esters had the highest linear correlation with the cube root of fruit weight $\left(R^{2}=0.7694\right)$, followed by the number of different aldehydes $\left(R^{2}=\right.$ $0.63223)$ and the number of total volatile compounds $\left(R^{2}=\right.$ 0.3793). Furthermore, Pearson's correlation analysis showed that the cube root of fruit weight exhibited a significant positive correlation with the number of types of esters $(r=0.877, P \leq$ 0.01 ), a significant negative correlation with the number of aldehydes $(r=-0.795, P \leq 0.01)$, a significant positive correlation with the number of total volatile compounds $(r=$ $0.616, P \leq 0.05$ ), and was not correlated with the number of alcohols, terpenes, and hydrocarbons (Table 4). Therefore, fruit weight correlates with the number of different types of esters, aldehydes, and total volatile compounds produced.

PCA OF THE VOLATILE COMPOUND CONTENTS. To decipher the role of volatile compounds in apple evolution, we analyzed the contents of alcohol compounds, ester compounds, aldehyde compounds, terpene compounds, and the total volatile compounds using PCA. The PCA results of alcohol compounds, ester compounds, and the total volatile compounds are shown in Fig. 4. Principal component 2 explained 18.10\% of the total variance observed and separated $M$. baccata and M. prunifolia into two groups according to the total volatile 


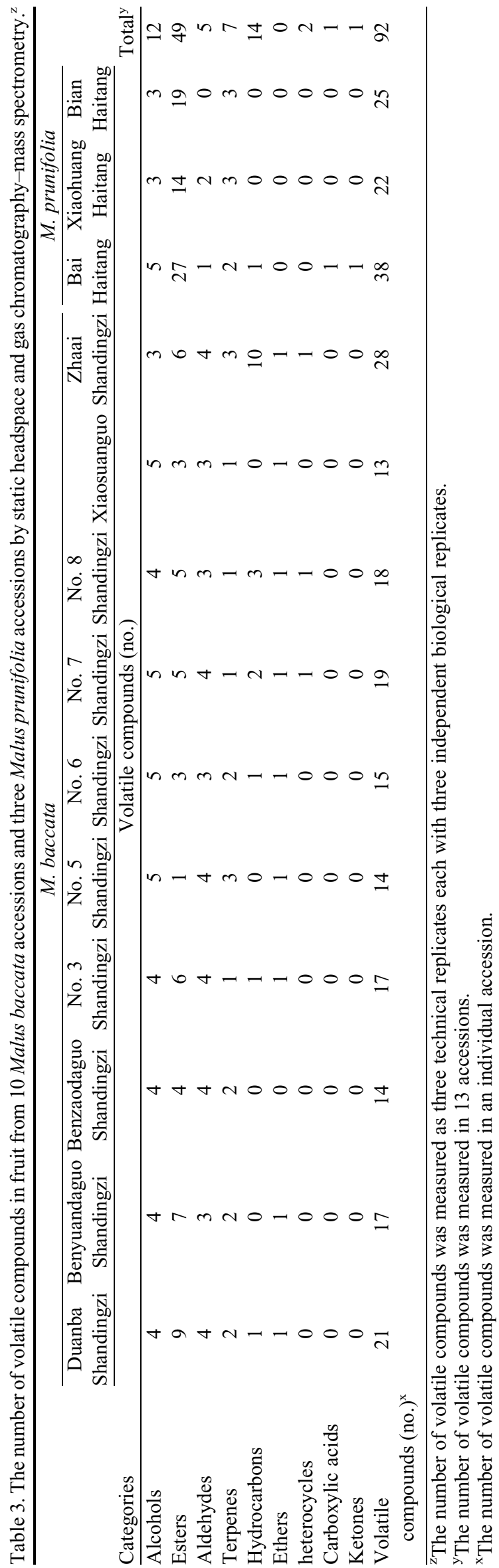

compound contents of apple species. The ester contents also separated M. baccata and M. prunifolia into two groups according to apple species, with three $M$. prunifolia accessions being closely clustered. However, alcohol contents could not be used to distinguish between $M$. baccata and M. prunifolia species.

\section{Discussion}

In this study, we examined the volatile compound profiles of $10 M$. baccata accessions and three M. prunifolia accessions using GC-MS. Malus baccata and M. prunifolia are two coldresistant species that are widely used for hardy rootstocks in northern China. 'Zhaai Shandingzi', a M. baccata accession, is an excellent dwarf hardy rootstock (the dwarf form is a descendant of the tall form) (Kuznetsova et al., 2010; Meng et al., 1997). We showed that 'Zhaai Shandingzi' was the $M$. baccata accession with the highest diversity of volatile compounds, having 28 different types of volatile compounds. Therefore, 'Zhaai Shandingzi' will be an excellent resource for enhancing the flavor of dwarf hardy apple. The $M$. prunifolia accession 'Bai Haitang' is widely distributed in northern China; due to its nice flavor and high level of cold resistance, it is used both for edible purposes and as a hardy rootstock. Simple sequence repeat marker analysis showed that 'Bai Haitang' was more distantly related to popular commercial cultivars (such as Golden Delicious, Jonathan, and Red Delicious) than to wild accessions, according to an unweighted pair-group method with arithmetic means dendrogram and principal coordinate analysis (Zhang et al., 2012). In our study, GC-MS analysis showed that 'Bai Haitang' produced 38 volatile compounds and had the highest diversity of volatile compounds of all M. prunifolia accessions. Therefore, 'Bai Haitang' will be an excellent resource for enhancing the flavor and widening the genetic diversity of commercial cultivars of apple.

Terpenes are compounds produced by plants that ward off pathogens and herbivores (Kessler and Baldwin, 2001). $\alpha$-Farnesene, a lepidopteran attractant and oviposition inducer (Bengtsson et al., 2001; Landolt et al., 2000; Šobotník et al., 2008; Sutherland and Hutchins, 1972), is the predominant terpene volatile produced during apple fruit storage (Huelin and Murray, 1966; Meigh and Filmer, 1969). However, the oxidation products of $\alpha$-farnesene can induce superficial scald disorder, which occurs in apple and pear fruit after several months of cold storage (Huelin and Coggiola, 1970; Isidoro and Almeida, 2006; Rowan et al., 2001). Wild apples have higher contents of terpene compounds than do domesticated cultivars (Chen et al., 2007; Wang et al., 2014). In our study, we found that M. prunifolia had a higher volatile terpene content than $\operatorname{did} M$. baccata, and that $\alpha$-farnesene was the most abundant terpene in all accessions examined. M. prunifolia fruit are difficult to store and are not commonly used for edible purposes. They are used as a prominent ornamental plant and robust rootstock for developing new apple cultivars (Igarashi et al., 2002). M. prunifolia may also be used in efforts to breed new apple cultivars with higher volatile terpene content.

Esters are the most abundant volatile compound in ripening apple fruit and normally account for $80 \%$ to $95 \%$ of the total volatile emission (Kakiuchi et al., 1986; Paillard, 1990). Sugimoto et al. (2015) evaluated ripening apple fruit from 184 germplasm lines, and found that ester diversity tended to 

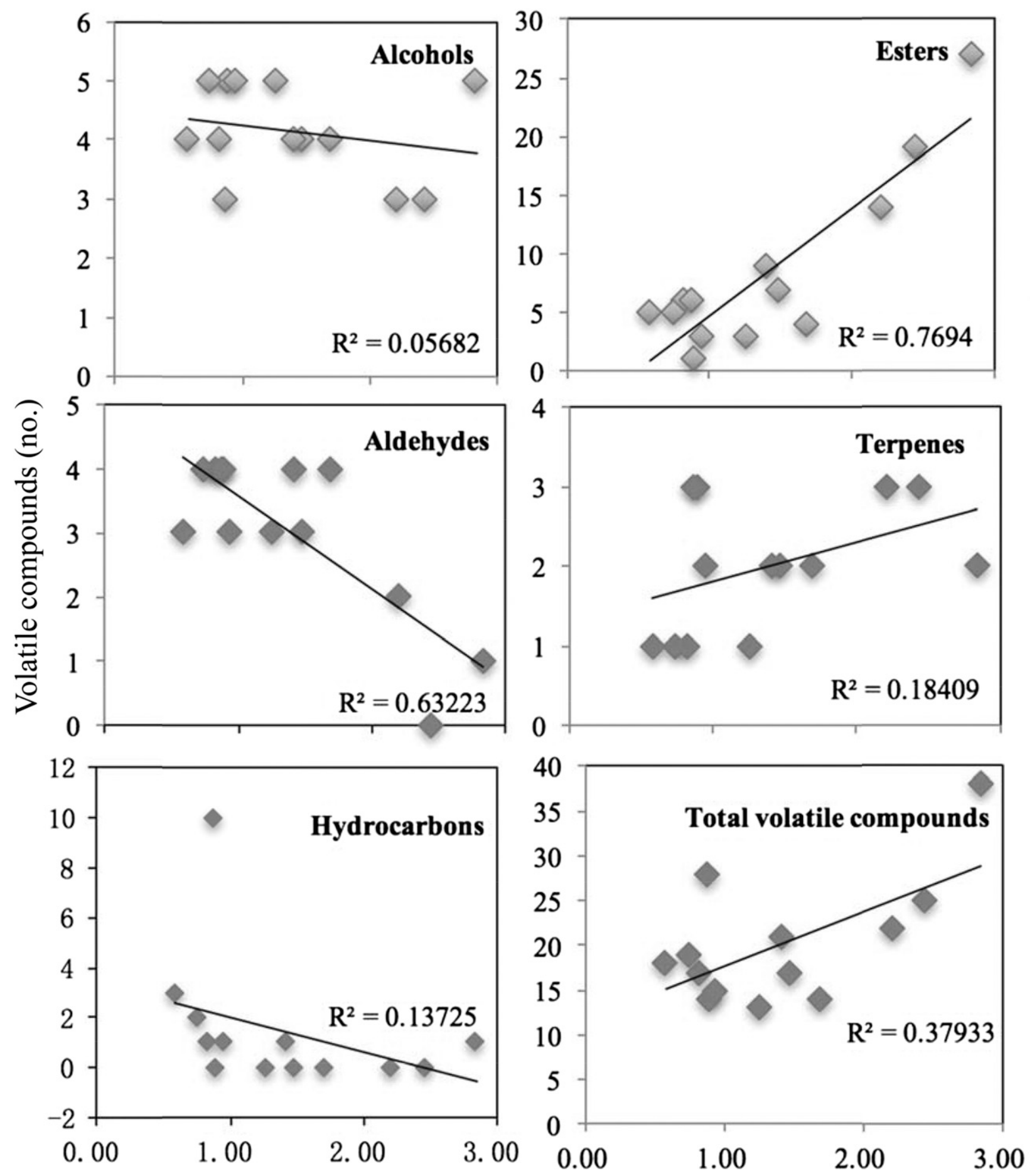

\section{Cube root of fruit weight $\left(\mathrm{g}^{-3}\right)$}

Fig. 3. Regression analysis between number of volatile compounds and the cube root of ripe fruit weight from 10 Malus baccata accessions ('Duanba Shandingzi', 'Xiaosuanguo', 'Zhaai Shandingzi', 'Benyuandaguo Shandingzi', 'Benzaodaguo Shandingzi', 'No. 3 Shandingzi', 'No. 5 Shandingzi', 'No. 6 Shandingzi', 'No. 7 Shandingzi', and 'No. 8 Shandingzi') and three Malus prunifolia accessions ('Bai Haitang', 'Xiaohuang Haitang', and 'Bian Haitang'). Volatile compounds were detected by static headspace and gas chromatography-mass spectrometry from ripe fruit as three technical replicates each with three independent biological replicates. Fruit weight was average weight of 30 fruit.

increase with increasing fruit weight and was linearly correlated with the cube root of fruit weight $\left(R^{2}=0.64\right)$. Our study showed that esters (49 kinds) accounted for $53.26 \%$ of the total volatile compounds ( 92 kinds) produced by wild apple and that ester diversity was more linearly correlated with the cube root of fruit weight $\left(R^{2}=0.7694\right)$ than was the total volatile compound diversity $\left(R^{2}=0.3793\right)$. Pearson's correlation analysis showed that the cube root of fruit weight 
Table 4. Pearson's correlation coefficients $(r)$ comparing the number of different types of volatile compounds and the cube root of the weight of an individual ripe fruit from 10 Malus baccata accessions and three Malus prunifolia accessions. ${ }^{2}$

\begin{tabular}{lllllll}
\hline & Alcohols & Esters & Aldehydes & Terpenes & Hydrocarbons & Total volatile compounds \\
\cline { 2 - 6 } & \multicolumn{5}{c}{ Pearson's correlation coefficients } \\
\hline Cube root of fruit weight $\left(\mathrm{g}^{-3}\right)$ & -0.238 & $0.877^{* * \mathrm{y}}$ & $-0.795^{* *}$ & 0.429 & -0.370 & $0.616^{*}$
\end{tabular}

$\overline{\mathrm{z}}$ Volatile compounds were detected by static headspace and gas chromatography-mass spectrometry from ripe fruit. Fruit weight was average weight of 30 fruit. Ten M. baccata accessions ('Duanba Shandingzi', 'Xiaosuanguo', 'Zhaai Shandingzi', 'Benyuandaguo Shandingzi', 'Benzaodaguo Shandingzi', 'No. 3 Shandingzi', 'No. 5 Shandingzi', 'No. 6 Shandingzi', 'No. 7 Shandingzi', and 'No. 8 Shandingzi') and three M. prunifolia accessions ('Bai Haitang', 'Xiaohuang Haitang', and 'Bian Haitang') were used to calculate Pearson's correlation coefficients.

${ }^{\text {y}}$ Pearson's correlation coefficient values with $*$ and $* *$ are significant differences by $t$ test at $P \leq 0.05$ and $\leq 0.01$, respectively.
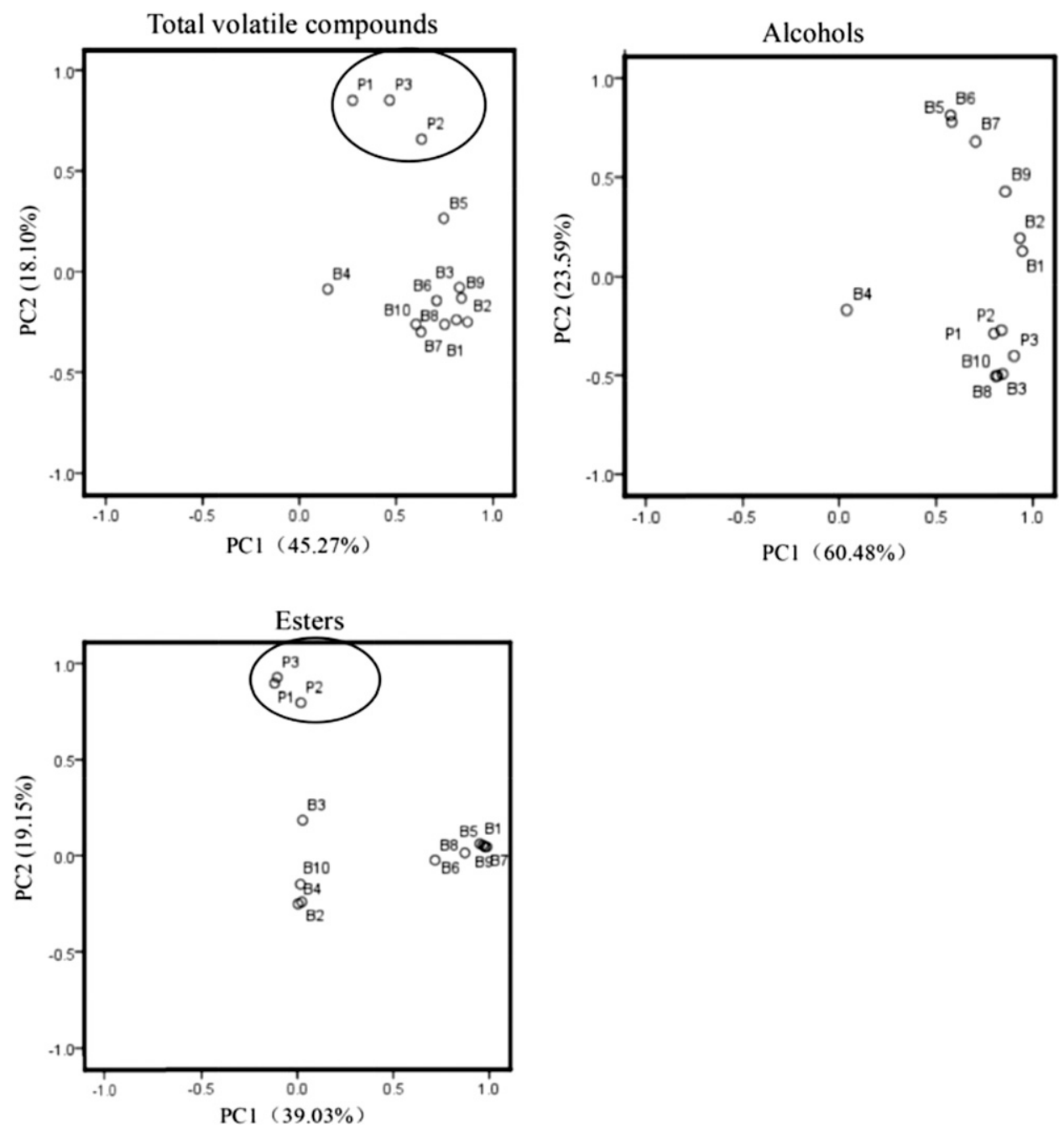

Fig. 4. Principal component (PC) analysis of the volatile compound contents. The first two principal components are shown (PC1 and PC2). Volatile compound contents were measured by static headspace and gas chromatography-mass spectrometry from ripe fruit as three technical replicates each with three independent biological replicates. Malus baccata accessions are abbreviated as follows: B1 = 'Duanba Shandingzi', B2 = 'Benyuandaguo Shandingzi', B3 = 'Benzaodaguo Shandingzi', B4 = 'No. 3 Shandingzi', B5 = 'No. 5 Shandingzi', B6 = 'No. 6 Shandingzi', B7 = 'No. 7 Shandingzi', B8 = 'No. 8 Shandingzi', B9 = 'Xiaosuanguo', and $\mathrm{B} 10=$ 'Zhaai Shandingzi'; Malus prunifolia accessions are abbreviated as follows: $\mathrm{P} 1=$ 'Bai Haitang', $\mathrm{P} 2=$ 'Xiaohuang Haitang', and P3 = 'Bian Haitang'. The two species were separated into two populations at the second principal component according to total volatile compound contents or ester contents. The circle meant that three M. prunifolia accessions were clustered together.

was more significantly positively correlated with ester diversity $(r=0.877, P \leq 0.01)$ than with total volatile compound diversity $(r=0.616, P \leq 0.05)$. Furthermore, PCA showed that the ester compound contents could better separate M. baccata and M. prunifolia accessions into two groups than could total volatile compound contents. Therefore, to expand the genetic diversity of commercial cultivars, ester compounds are important indicators of parental choice in apple breeding. At the same time, consumers perceived esters (hexyl acetate, butyl acetate, and 2-methylbutyl acetate) as "fruity" and "floral" (Aharoni et al., 2000; Plotto et al., 2000). Our study showed that hexyl acetate was detected in most M. baccata accessions and M. prunifolia accession 'Xiaohang Haitang'. Therefore, these accessions might be good breeding materials to meet the sensory needs of consumers.

\section{Literature Cited}

Aharoni, A., L.C.P. Keizer, H.J. Bouwmeester, Z. Sun, M. AlvarezHuerta, H.A. Verhoeven, J. Blass, A.M.M.L. van Houwelingen, R.C.H. De Vos, H. van der Voet, R.C. Jansen, M. Guis, J. Mol, R.W. Davis, M. Schena, A.J. van Tunen, and A.P. O'Connell. 2000. Identification of the SAAT gene involved in strawberry flavor biogenesis by use of DNA microarrays. Plant Cell 12:647-661.

Bengtsson, M., A.C. Bäckman, I. Liblikas, M.I. Ramirez, A.K. Borg-Karlson, L. Ansebo, P. Anderson, J. Löfqvist, and P. Witzgall. 2001. Plant odor analysis of apple: Antennal response of codling moth females to apple volatiles during phenological development. J. Agr. Food Chem. 49: 3736-3741.

Chen, X., T. Feng, Y. Zhang, T. He, J. Feng, and C. Zhang. 2007. Genetic diversity of volatile components in Xinjiang wild apple (Malus sieversii). J. Genet. Genomics 34:171-179.

Dimick, P.S. and J.C. Hoskin. 1983. Review of apple flavor: State of the art. Crit. Rev. Food Sci. Nutr. 18:387-409.

Emeriewen, O., K. Richter, A. Kilian, E. Zini, M. Hanke, M. Malnoy, and A. Peil. 2014. Identification of a major quantitative trait locus for 
resistance to fire blight in the wild apple species Malus fusca. Mol. Breed. 34:407-419.

Forsline, P.L., H.S. Aldwinckle, E.E. Dickson, J.J. Luby, and S.C. Hokanson. 2010. Collection, maintenance, characterization, and utilization of wild apples of central Asia. Hort. Rev. 29:1-61.

Huelin, F.E. and I.M. Coggiola. 1970. Superficial scald, a functional disorder of stored apples. VII: Effect of applied $\alpha$-farnesene, temperature, and diphenylamine on scald and the concentration and oxidation of $\alpha$-farnesene in the fruit. J. Sci. Food Agr. 21:584-589.

Huelin, F.E. and K.E. Murray. 1966. $\alpha$-Farnesene in the natural coating of apples. Nature 210:1260-1261.

Igarashi, M., H. Ogasawara, Y. Hatsuyama, A. Saito, and M. Suzuki. 2002. Introduction of rolC into Marubakaidou (Malus prunifolia Borkh. var. ringoAsami Mo 84-A) apple rootstock via Agrobacterium tumefaciens. Plant Sci. 163:463-473.

Isidoro, N. and D.P.F. Almeida. 2006. $\alpha$-Farnesene, conjugated trienols, and superficial scald in 'Rocha' pear as affected by 1methylcyclopropene and diphenylamine. Postharvest Biol. Technol. 42:49-56.

John, K.M.M., G. Enkhtaivan, J.J. Kim, and D.H. Kim. 2014. Metabolic variation and antioxidant potential of Malus prunifolia (wild apple) compared with high flavon-3-ol containing fruits (apple, grapes) and beverage (black tea). Food Chem. 163:46-50.

Jurick, W.M., W.J. Janisiewicz, R.A. Saftner, I. Vico, V.L. Gaskins, E. Park, P.L. Forsline, G. Fazio, and W.S. Conway. 2011. Identification of wild apple germplasm (Malus spp.) accessions with resistance to the postharvest decay pathogens Penicillium expansum and Colletotrichum acutatum. Plant Breed. 130:481-486.

Kakiuchi, N., S. Noriguchi, H. Fukuda, N. Ichimura, Y. Kato, and Y. Banba. 1986. Composition of volatile compounds of apple fruits in relation to cultivars. J. Jpn. Soc. Hort. Sci. 55:280-289.

Kessler, A. and I.T. Baldwin. 2001. Defensive function of herbivoreinduced plant volatile emissions in nature. Sci. 291:2141-2144.

Kumar, S., R. Volz, P. Alspach, and V. Bus. 2010. Development of a recurrent apple breeding programme in New Zealand: A synthesis of results, and a proposed revised breeding strategy. Euphytica 173:207-222.

Kuznetsova, E.V., T.E. Peretolchina, A.V. Rudikovskii, and D.Y. Sherbakov. 2010. Speciation in status nascendi in siberian crabapple Malus backata (L.). Borkh. Moscow Univ. Biol. Sci. Bul. 65:196-199. Landolt, P.J., J.A. Brumley, C.L. Smithhisler, L.L. Biddick, and R.W. Hofstetter. 2000. Apple fruit infested with codling moth are more attractive to neonate codling moth larvae and possess increased amounts of E,E-alpha farnesene. J. Chem. Ecol. 26:1685-1699.

Laurens, F. 1999. Review of the current apple breeding programmes in the world: Objectives for scion cultivar improvement. Acta Hort. 484:163-170.

Li, X., L. Kang, J. Hu, X. Li, and X. Sheng. 2008. Aroma volatile compound analysis of SPME headspace and extract samples from crabapple (Malus sp.) fruit using GC-MS. Agr. Sci. China 7:1451-1457.

Li, Y., Y. Tan, Y. Shao, M. Li, and F. Ma. 2015. Comprehensive genomic analysis and expression profiling of diacylglycerol kinase gene family in Malus prunifolia (Willd.). Borkh. Gene 561:225-234. Mattheis, J.P., D.A. Buchanan, and J.K. Fellman. 1991. Change in apple fruit volatiles after storage in atmospheres inducing anaerobic metabolism. J. Agr. Food Chem. 39:1602-1605.
Meigh, D.F. and A.A.E. Filmer. 1969. Natural skin coating of the apple and its influence on scald in storage. III. $\alpha$-farnesene. J. Sci. Food Agr. 20:139-143.

Meng, Q.Y., X.G. Wang, N. Ta, S.M. Yuan, Z.X. Gong, and R.H. Zhou. 1997. Dwarf hardy germplast of apple Malus baccata - Zhaai Shandingzi. China Fruits 3:13-14.

Noiton, D.A.M. and P.A. Alspach. 1996. Founding clones, inbreeding, coancestry, and status number of modern apple cultivars. J. Amer. Soc. Hort. Sci. 121:773-782.

Paillard, N.M.M. 1990. The flavour of apples, pears and quinces, p. 1-41. In: I.D. Morton and A.J. Mcleod (eds.). Food flavours. Part C. The flavour of fruits. Elsevier, Amsterdam, The Netherlands.

Plotto, A., M.R. McDaniel, and J.P. Mattheis. 2000. Characterization of changes in 'Gala' apple aroma during storage using Osme analysis, a gas chromatography-olfactometry technique. J. Amer. Soc. Hort. Sci. 125:714-722.

Rowan, D.D., M.B. Hunt, S. Fielder, J. Norris, and M.S. Sherburn. 2001. Conjugated triene oxidation products of alpha-farnesene induce symptoms of superficial scald on stored apples. J. Agr. Food Chem. 49:2780-2787.

Scalzo, R.L., A. Testoni, and A. Genna. 2001. 'Annurca' apple fruit, a southern Italy apple cultivar: Textural properties and aroma composition. Food Chem. 73:333-343.

Sedov, E.N. and Z.M. Serova. 2013. Apple breeding for the high commercial and consumer appeal of fruit. Russ. Agr. Sci. 39:241244.

Šobotník, J., R. Hanus, B. Kalinová, R. Piskorski, J. Cvaêka, T. Bourguignon, and Y. Roisin. 2008. (E,E)- $\alpha$-Farnesene, an alarm pheromone of the termite Prorhinotermes canalifrons. J. Chem. Ecol. 34:478-486.

Sugimoto, N., P. Forsline, and R. Beaudry. 2015. Volatile profiles of members of the USDA Geneva Malus core collection: Utility in evaluation of a hypothesized biosynthetic pathway for esters derived from 2-methylbutanoate and 2-methylvutan-1-ol. J. Agr. Food Chem. 63:2106-2116.

Sutherland, O.R.W. and R.F.N. Hutchins. 1972. $\alpha$-Farnesene, a natural attractant for codling moth larvae. Nature 239:170.

Wang, R., X. Shen, C. Wang, R. Ge, Z. Zhang, and X. Guo. 2014. Analysis of leaf volatiles of crabapple (Malus sp.) individuals in different aphids's resistance. Amer. J. Plant Sci. 5:3295-3301.

Wang, L., Z. Wang, and X. Li. 2013. Preliminary phytochemical and biological activities study of solvent extracts from a cold-field fruitMalus backata (Linn.). Borkh. Ind. Crops Prod. 47:20-28.

Zhang, B. 2008. Study on the genetic diversity of winter hardy apple and pear germplasm resources. Jilin Agr. Univ., Changchun, China, PhD Diss.

Zhang, C., X. Chen, T. He, X. Liu, T. Feng, and Z. Yuan. 2007. Genetic structure of Malus sieversii population from Xinjing, China, revealed by SSR markers. J. Genet. Genomics 34:947955.

Zhang, Q., J. Li, Y. Zhao, S.S. Korban, and Y. Han. 2012. Evaluation of genetic diversity in Chinese wild apple species with apple cultivars using SSR markers. Plant Mol. Biol. Rpt. 30:539-546.

Zhang, B., H. Liu, H. Song, and Y. Zhang. 2003. Investigation of fruit germplasm resources with cold resistance in Jilin province. China Acta Hort. 620:175-181. 


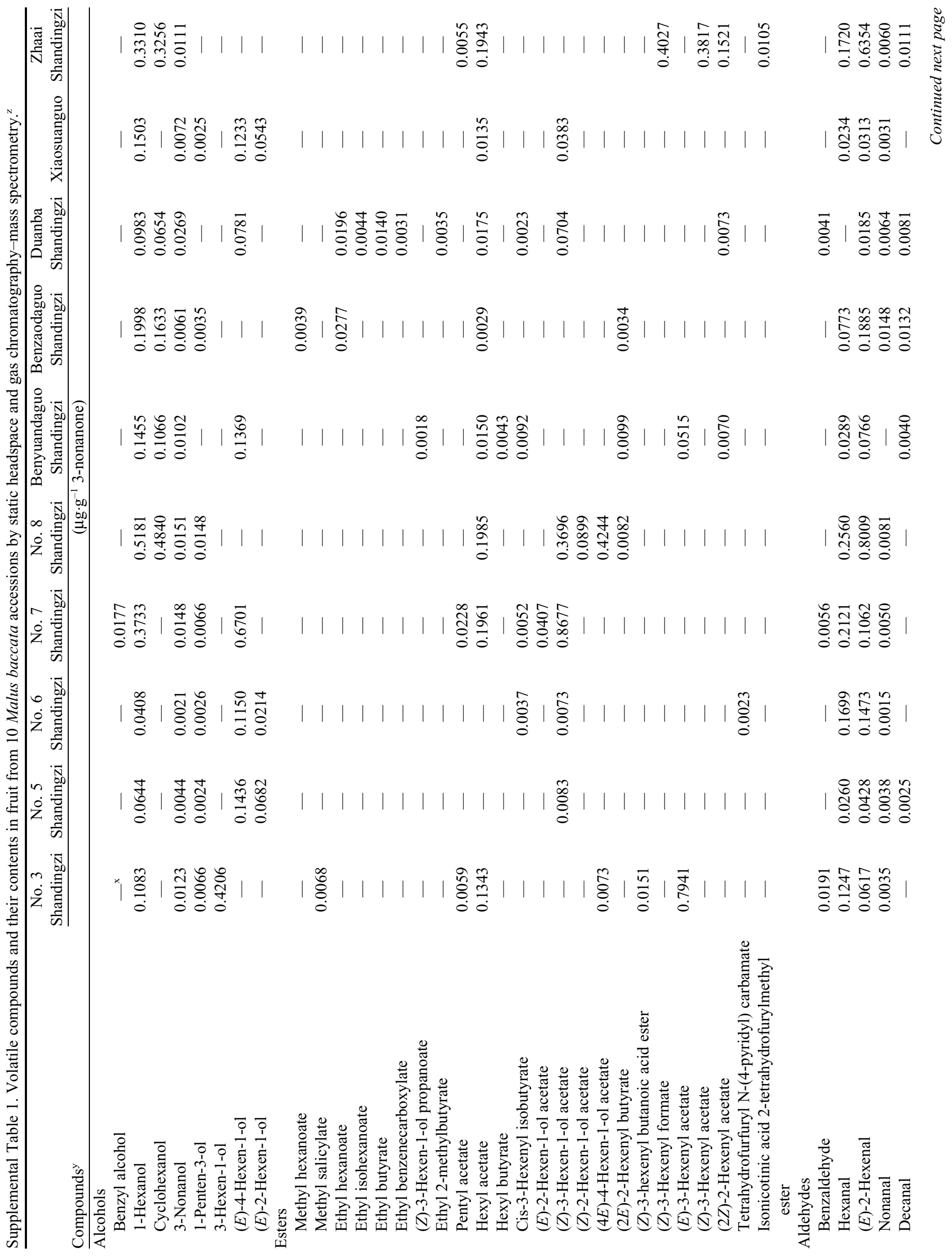




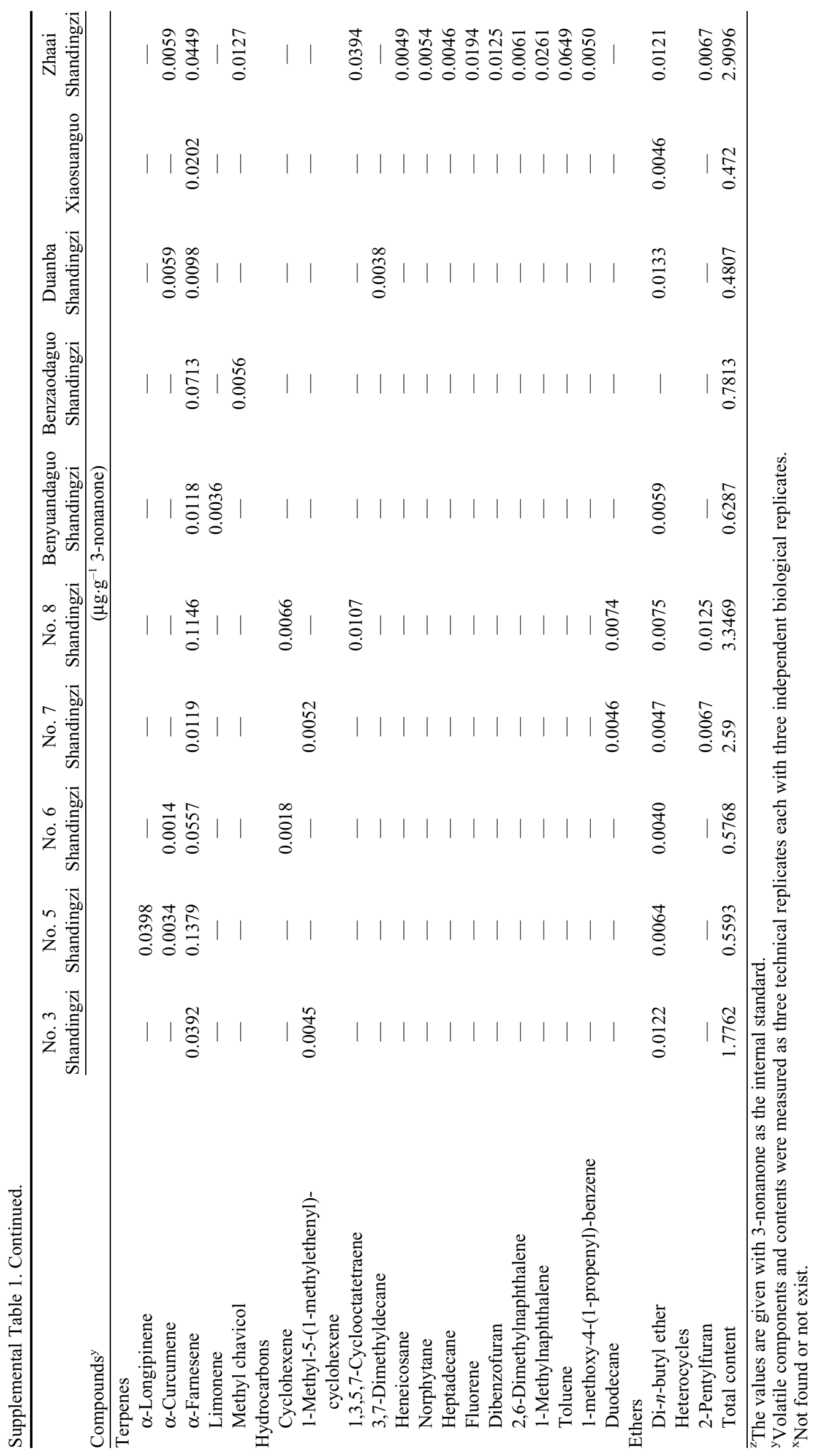


Supplemental Table 2. Volatile compounds and their contents in fruit from three Malus prunifolia accessions by static headspace and gas chromatography-mass spectrometry. ${ }^{z}$

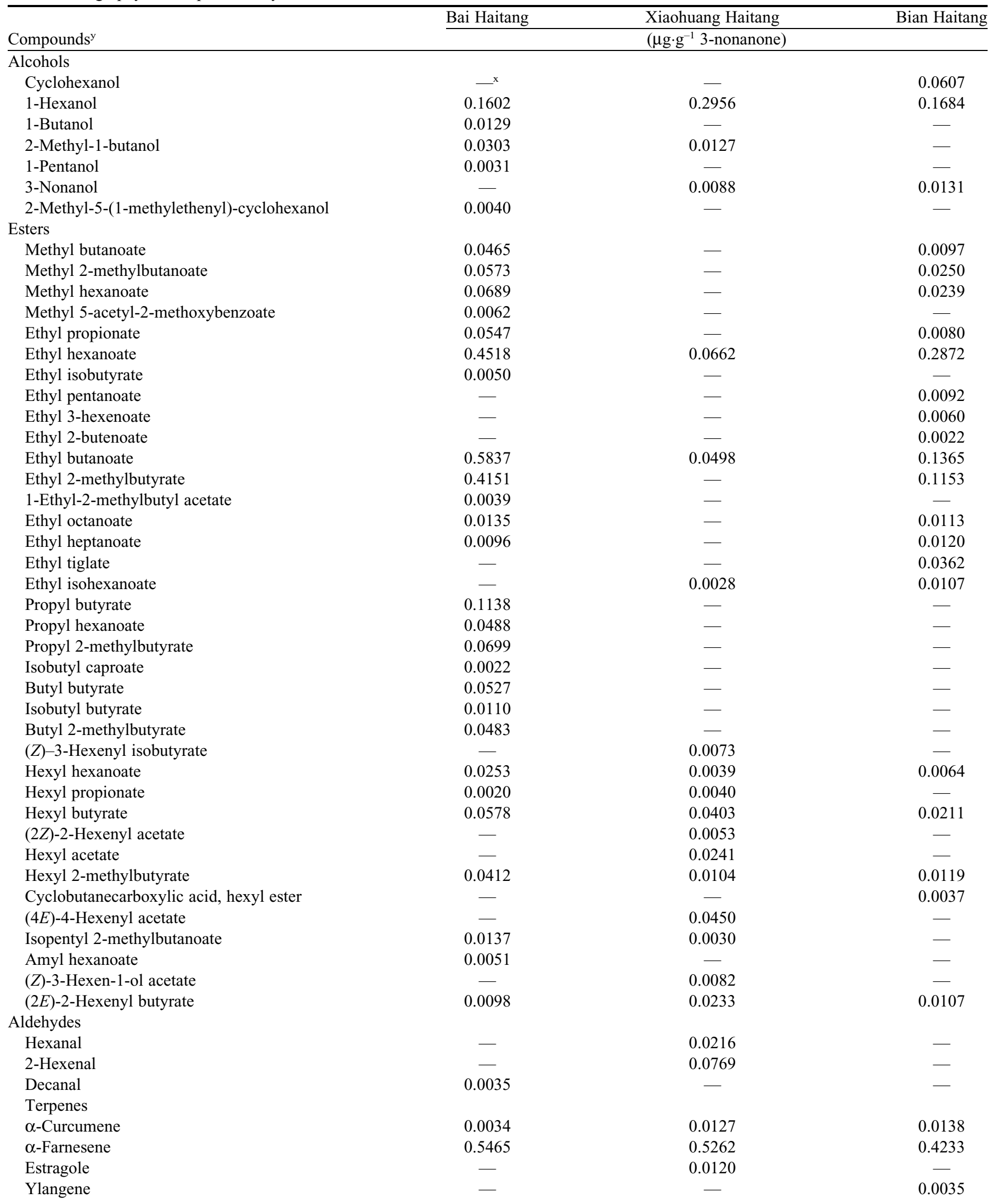


Supplemental Table 2. Continued.

\begin{tabular}{|c|c|c|c|}
\hline \multirow[b]{2}{*}{ Compounds ${ }^{\mathrm{y}}$} & Bai Haitang & Xiaohuang Haitang & Bian Haitang \\
\hline & \multicolumn{3}{|c|}{ ( $\mu \mathrm{g} \cdot \mathrm{g}^{-1}$ 3-nonanone) } \\
\hline \multicolumn{4}{|l|}{ Hydrocarbons } \\
\hline Fluorene & 0.0103 & - & - \\
\hline 6-Methyl-5-hepten-2-one & 0.0029 & - & - \\
\hline Carboxylic acids & & & \\
\hline
\end{tabular}

${ }^{\mathrm{z}}$ The values are given with 3-nonanone as the internal standard.

${ }^{\mathrm{y}}$ Volatile components and contents were measured as three technical replicates each with three independent biological replicates.

${ }^{\mathrm{x}}$ Not found or not exist. 